\title{
Nuclear Morphogenesis During the Developmental Cycles of Some Members of the Genus Nocardia
}

\author{
BY J. N. ADAMS* \\ Department of Bacteriology, University of Georgia, Athens, Georgia, U.S.A.
}

SUMMARY

Observations of nuclear morphogenesis were carried out using a modification of the acid hydrolysis-Giemsa technique. Throughout the development cycles studied, nuclear division was found to occur by means of elongation of the chromatin bodies with subsequent medial constriction and separation into daughter structures. Nuclear division greatly exceeded septum deposition during the early phases of the developmental cycles and, as a result, coenocytic filaments were formed. Fragmentation of these filaments resulted in the production of single cells, short chains and short coenocytes like those found in the inoculum. No evidence for complex nuclear fusions or reductional divisions was obtained during any period of nuclear morphogenesis and chromatin body divisions were considered to be amitotic. A simple model explaining previously reported genetic recombination in the genus Nocardia is presented.

\section{INTRODUCTION}

A recent investigation, concerned with cellular development from the standpoint of the behaviour of the cell wall during the growth of the organisms, elucidated the developmental cycles of selected members of the genus Nocardia (Adams \& McClung, $1962 a$ ). While earlier reports described nocardial nuclear morphogenesis, the results of these investigations were conflicting (McClung, 1950, 1955; Morris, 1951; Webb, Clark \& Chance, 1954; Webb \& Clark, 1957; Clark \& Frady, 1957; and Hagedorn, $1959 a, b)$ and many of the staining techniques used were unsuitable (Adams \& McClung, 1962 b). The discovery of genetic recombination in the Nocardiae (Adams \& Bradley, 1963) necessitates clarification of the nature of nocardial nuclear morphogenesis to help understand genetic interactions among the group. The present investigations employing a technique which has been demonstrated to be suitable for specific nuclear staining (Adams \& McClung, 1962b) were undertaken in order to describe nuclear activity in several species of the genus Nocardia.

\section{METHODS}

The strains of Nocardia rubra, N. erythropolis, N. canicruria and N. asteroides used in these studies were described previously (Adams \& McClung, 1962a). Nuclear staining was carried out with a modification of the Robinow (1945) technique which was shown in our laboratory (Adams \& McClung, 1962b) to be deoxyribonucleic-acid specific when applied to these organisms. Distilled water suspensions of 3-day-old Difco nutrient agar grown cultures were prepared and nutrient agar plates uniformly inoculated with the suspensions. After periods of incubation

* Present address : Department of Microbiology, Medical School, University of South Dakota, Vermillion, South Dakota, U.S.A. 
ranging from 1 to $72 \mathrm{hr}$ at $28^{\circ}$ blocks $c .1 \mathrm{~cm} .^{2}$, were cut from the agar and fixed for $1.5 \mathrm{~min}$. over the vapours of $2 \%(\mathrm{w} / \mathrm{v})$ aqueous osmium tetroxide. Impression smears were then prepared from the fixed blocks and the smears were hydrolysed for $6 \mathrm{~min}$. in $1 \mathrm{~N}-\mathrm{HCl}$ at $60^{\circ}$, washed with tap and distilled water and stained for 5 min. in a freshly prepared 1/10 dilution of Giemsa's solution in tap water. Excess Giemsa's solution was washed off with distilled water and the smear was blotted dry. Smears were examined directly; that is, were not mounted under a cover glass prior to observation. Photomicrography and observations were carried out with equipment described previously (Adams \& McClung, 1962b).

\section{RESULTS}

\section{Nocardia rubra nuclear development}

Cells composing the inoculum (Pl. 1, fig. 1) commonly contained single, spherical to ovoid chromatin bodies. During the first few hours incubation, the period in which cells appeared to remain unchanged when observed by means of cell-wall stained preparations (Adams \& McClung, 1962a), the chromatinic structures divided. Elongation of the deeply staining bodies was seen and after the chromatin bodies assumed a bar shape, a medial constriction appeared giving the dividing chromatin bodies a characteristic dumb-bell form (Pl. 1, fig. 2). The inclusions then separated into two distinct structures (Pl. 1, fig. 3). During the period from 5 to $8 \mathrm{hr}$., germ tube protrusions were produced and one of the chromatin bodies resulting from the first nuclear division migrated into the forming germ tube. The chromatin body in the germ tube elongated and continued divisional processes (Pl. 1, fig. 4) leading to the production of germ tubes (Pl. 1, fig. 5) containing many chromatin bodies appearing to be in various stages of division. The nuclei divided when in central positions as well as when located distal to the germinating cell. In the case of the production of more than one germ tube by a cell the process of chromatin body division was much like that described for cells producing single germ tubes. In these instances, nuclear divisions in the originating cells continued and migration of chromatin structures into the second as well as the first germ tube occurred.

Transition from the germination phase (Pl. 1, figs. 1-5) to that of mycelial development (Pl. 1, figs. 6-9) was relatively uneventful; chromatinic activity differing from that previously described was not revealed. Filaments continued to grow and chromatin behaviour in the filamentous cells (Pl. 1, figs. 6-9) was like that which occurred in the germ tubes.

The activity of chromatin bodies in cells producing a branch on the germ tube near the originating cell, a phenomenon first observed in cell-wall stained preparations (Adams \& McClung, 1962 a), was noted. Germ tube growth proceeded with concomitant chromatin body division (Pl. 1, fig. 10) and branches proximal to the originating cell (Pl. 1, figs. 11, 12) were produced. Chromatin bodies migrated (Pl. 1, fig. 11) into the branch and, after migration, the nuclei resumed observable divisional processes until the branches contained complements of chromatin bodies (Pl. 1, fig. 12).

In the case of primary branch formation, branches were produced at any point on the filament length (Pl. 1, figs. 9, 13, 14). It might be expected that chromatin 
activity is responsible for the location of a branch at a particular site on the filament, but there was no indication that branch growth began at a specific site in direct response to the presence of a chromatin body which could be differentiated from others because of configuration. However, Pl. 1, figs. 8, 9, and 11, indicate that chromatin bodies migrate into branches after they are formed. Frequently short, immature branches were found in which chromatin bodies could not be discerned with the technique used.

During the period of filament fragmentation the most noticeable change observed was the cessation of chromatin body division (Pl. 1, figs. 13-19). The elongated bar to dumb-bell shaped chromatinic structures, indicating active division (Pl. 1, figs. 1-12), became fewer although several could usually be seen in any specific filament (Pl. 1, figs. 13, 15). As fragmentation continued, nuclei became more remotely separated and readily distinguishable as entities (Pl. 1, figs. 14-17). During the period in which filament length dramatically decreased (Pl. 1, figs. 18, 19), a result of septum formation and subsequent separation at the septa (Adams \& McClung, 1962a), the distinct chromatin bodies underwent little apparent activity and did not appear to divide again. Consequently, the greatest part of the population during this period of 32-36 hr. incubation consisted of short filament fragments and individual cells which were similar in chromatin body complement to those found in the inocula.

\section{Nuclear morphogenesis in other Nocardiae}

Morphogenesis of chromatin bodies during the developmental cycles of Nocardia erythropolis was essentially identical to that observed in N. rubra. Most cells in the inoculum contained single, discrete chromatin bodies although short coenocytes and chains of cells were frequently observed. During early incubation (Pl. 2, fig. 20) chromatin bodies elongated and divided. As filaments increased in length (Pl. 2, figs. 21-23) chromatin body division occurred in both median (Pl. 2, fig. 21) and terminal positions (Pl. 2, fig. 22). Nuclear migration into old (Pl. 2, fig. 22) and new branches (Pl. 2, fig. 23) was commonly observed but definitive chromatin body configuration could not be correlated with branch location. Cessation of nuclear division and fragmentation was like that observed in $N$. rubra.

Nuclear morphogenesis of Nocardia canicruria was essentially like that observed in $N$. rubra and $N$. erythropolis but the developmental cycle was considerably shorter, being $24 \mathrm{hr}$. as compared to $36 \mathrm{hr}$. or more for $N$. rubra and $30 \mathrm{hr}$. for $N$. erythropolis. Germination, nuclear migration and division was like that of the other Nocardiae examined (Pl. 2, fig. 24). During the period of mycelial development, 9-18 hr. incubation (Pl. 2, figs. 25-27), an interesting phenomenon was noted; relative synchrony of division of chromatin bodies could be observed in the filaments. Frequently all of the chromatin bodies in a single filament were dividing (P1. 2, fig. 26) and were apparently in the same stage of the division process. Such synchronous chromatin body division was not commonly observed in any of the other organisms studied. However, fragmentation was like that previously noted (Pl. 2, fig. 28).

The nuclear development of Nocardia asteroides was in all respects like that found in $N$. rubra and $N$. erythropolis but chromatin body division synchrony like that of $N$. canicruria was not observed. The cycle in $N$. asteroides encompassed a $72 \mathrm{hr}$. span. Nuclear division in germinating single cells as well as short hyphal fragments 
(Pl. 2, fig. 29) was seen. Mycelial development, in which branching filaments were observed with complements of dividing chromatin bodies (Pl. 2, fig. 30), was more extensive than in the other organisms studied. Cessation of chromatin body divisions during fragmentation was like that observed in the other Nocardiae. The degree of septation and separation at the septa determined the relative numbers of uni-nucleate cells and short coenocytes present at the completion of fragmentation.

\section{DISCUSSION}

Using killed and fixed preparations, it is not possible to observe directly the fate of individual chromatin bodies or to determine whether dumb-bell- and bar-shaped structures are the result of fusion of two separate nuclei or a consequence of division of one chromatin body into daughter structures. Observation of nuclear morphogenesis in living cells, employing phase microscopy and the technique of Mason \& Powelson (1956) which allow resolution of this problem, is not possible. Metachromatic granules are abundant in the Nocardiae and differentiation of these granules from chromatin structures depends upon cytochemical specificity (Adams $\&$ McClung, 1962b). However, several observations direct a choice between the alternatives of the occurrence of nuclear fusions or divisions during early developmental stages of the Nocardiae. Newly formed germ tubes and branches, indicated by relatively short lengths, frequently are devoid of chromatin bodies. Slightly longer germ tubes or branches contain single spherical structures. Slightly longer germ tubes or branches contain bar and dumb-bell chromatin bodies. Since these are found in longer, older branches and germ tubes, it follows that dumb-bell and bar-shaped structures arise as a consequence of divisional processes after nuclear migration.

While the above hypothesis accounts for the behaviour of chromatin bodies during the early period of nocardial development, it may not explain nuclear activity observed during fragmentation. Several estimates of the number of chromatin bodies found in filaments during the early phases of fragmentation, in which the dumb-bell or bar forms were counted as two, rather than one nucleus, indicate that filaments containing such structures have a chromatin body complement per unit cell length approximately equivalent to that of cells and coenocytes appearing in the inoculum or at the termination of fragmentation. Nuclear behaviour based on a process involving fusion and subsequent division of fused nuclei during this period would demand the presence of a complement of two chromatin bodies per unit cell length at this stage compared to the terminal phase of fragmentation and unabated nuclear activity. Neither was observed. Morris (1951) described a complex life cycle, including several nuclear fusion and reductional division stages, for members of the genus Nocardia. His report, undocumented with micrographs, in light of the work of others (McClung, 1950, 1955; Webb et al. 1954; Webb \& Clark, 1957; and Hagedorn, $1959 a, b)$ and the present observations, lead the author to discount the complex life cycle he described. Webb et al. observed discrete nuclei in most phases of the growth of Nocardia corallina. A more detailed study (Webb \& Clark, 1957) indicated that a developmental cycle involving complex nuclear morphogenesis exists in $N$. corallina. However, Hagedorn (1959a) suggested that the results of Webb \& Clark (1957) were obtained with an inadequate nuclear staining technique. 
The work of Adams \& McClung (1962 b) has shown that the stain which they (Webb \& Clark, 1957) employed was not satisfactory for proper identification of nuclear material in some instances. On such bases, it seems likely that the complex stages they observed were caused by the staining technique rather than an effect brought about by cellular changes in the organisms.

Although nuclear morphogenesis in the Nocardiae does not appear to involve complex cyclic reductional division stages, it cannot be concluded that the morphologically simple, amitotic-like process observed in the present studies is insignificant. Previous studies in our laboratory (Adams \& McClung, 1962a) have shown that Nocardia erythropolis and N. canicruria are morphologically closely related organisms. The observations presented in this report further support this supposition since nuclear morphogenesis in the two species, while not exactly identical, is similar. Such similarities take on greater significance when it is recognized that interactions of a genetic recombinational nature occur in mixed cultures of mutants of these two organisms (Adams \& Bradley, 1963).

This study does not purport to explain definitively the mechanism of genetic recombination events which have been reported in the genus Nocardia. However, on the basis of the morphological findings reported herein, a mechanical model for this genetic phenomenon can be offered. The demonstration of coenocytic filaments substantiates the possibility that after hyphal anastomosis (Adams \& McClung, $1962 a$ ) nuclei of diverse origin could interact within a common cytoplasm. In this manner, genetically defined heterokaryosis (Bradley \& Lederberg, 1956) or synkaryosis (Sermonti \& Spada-Sermonti, 1956; and Braendle \& Szybalski, 1957) like that occurring in the streptomycetes could be initiated. The Nocardiae, not unlike other organisms in this respect, have been shown in the present investigations to produce cells which contain single chromatin bodies. This nuclear segregation could also be expected to segregate genetically determined characters residing in the nucleus. Hence cytologically described nuclear morphogenesis offers a mechanism for both intergenomic action as well as segregation of recombinant genomes. However, cytological observations of the Nocardiae, even with the aid of electron microscopy of ultra-thin sections (Hagedorn, 1959a,b) do not offer conclusive evidence regarding ploidy or complex nuclear reduction divisions, but they do offer a simple model explaining genetic interactions among these organisms. It is felt that further genetic studies of the Nocardiae, which are now in progress, may alternatively serve to offer a basis for a clearer interpretation of nuclear morphogenesis in this group.

The author is indebted to N. M. McClung for his helpful suggestions and frank criticisms throughout the course of these investigations. This study was made possible by grant E-2075 from the National Institute of Allergy and Infectious Diseases, U.S. Public Health Service, to N. M. McClung.

\section{REFERENCES}

Adams, J. N. \& Bradley, S. G. (1963). Recombination events in the bacterial genus Nocardia. Science, 140, 1392.

Adams, J. N. \& McClung, N. M. (1962a). Comparison of the developmental cycles of some members of the genus Nocardia. J. Bact. 84, 206.

Adams, J. N. \& McClung, N. M. (1962b). On the nature of cytoplasmic inclusions of Nocardia rubra. J. gen. Microbiol. 28, 231. 
Bradley, S. G. \& Lederberg, J. (1956). Heterokaryosis in streptomyces. J. Bact. 72, 219.

Braendle, D. H. \& Szybalski, W. (1957). Genetic interaction among streptomycetes: heterokaryosis and synkaryosis. Proc. nat. Acad. Sci., Wash. 43, 947.

Clark, J. B. \& Frady, J. (1957). Secondary life cycle of Nocardia corallina. J. Bact. 74, 698.

HAGEdoRn, H. (1959a). Licht- und elektronenmikroskopische Untersuchungen an Nocardia corallina (Bergey et al. 1923). Z Zbl. Bakt. Abt. 2, 112, 214.

HagedonN, H. (1959b). Elektronenmikroskopische Untersuchungen über den Teilungsverlauf bei Nocardia corallina (Bergey et al. 1923). Zbl. Bakt. Abt. 2, 112, 359.

Mason, D. J. \& Powelson, D. M. (1956). Nuclear division as observed in live bacteria by a new technique. J. Bact. 71, 474.

McClung, N. M. (1950). Morphological studies in the genus Nocardia. II. Cytological studies. J. Bact. 59, 589.

MCClung, N. M. (1955). Morphological studies in the genus Nocardia. IV. Bright phase contrast observations of living cells. Trans. Kans. Acad. Sci. 58, 50.

Morris, E. O. (1951). Observations on the life cycle of the Nocardia. J. Hyg., Camb. 49, 175.

RoBinow, C. F. (1945). Nuclear apparatus and cell structure of rod-shaped bacteria. In Addendum to The Bacterial Cell, by R. J. Dubos. Cambridge, Mass. : Harvard University Press.

Sermonti, G. \& Spada-Sermonti, I. (1956). Gene recombination in Streptomyces coelicolor. J. gen. Microbiol. 15, 609.

WebB, R. B. \& Clark, J. B. (1957). Cytogenetic study of Nocardia corallina. J. Bact. 74, 31.

Webe, R. B., Clark, J. B. \& Chance, H. L. (1954). A cytological study of Nocardia corallina and other actinomycetes. J. Bact. 67, 498.

\section{EXPLANATION OF PLATES}

Figs. 1-30. Robinow's acid Giemsa stained cells. All micrographs $\times \mathbf{2 5 0 0}$.

Figs. 1-19. Nocardia rubra.

Plate 1

Fig. 1. Inoculum.

Figs. 2, 3. $2 \mathrm{hr}$. culture.

Fig. $4.5 \mathrm{hr}$. culture.

Fig. 5. $7 \mathrm{hr}$. culture.

Fig. 6. $9 \cdot 5 \mathrm{hr}$. culture; arrows indicate chromatin body division in medial positions.

Fig. 7. 9.5 hr. culture.

Fig. $8.17 \mathrm{hr}$. culture; arrow indicates single spherical chromatin body in branch.

Fig. 9. $17 \mathrm{hr}$. culture; arrow indicates a dividing chromatin body in branch.

Fig. 10. $17 \mathrm{hr}$. culture.

Fig. 11. $19 \mathrm{hr}$. culture. Arrow indicates chromatin bodies in process of migrating into branch.

Fig. 12. $17 \mathrm{hr}$. culture.

Figs. 13-15. $25 \mathrm{hr}$. cultures. Arrows indicate actively dividing chromatin bodies.

Figs. 16-17. 26.5 hr. culture.

Figs. 18, 19. $32 \mathrm{hr}$. culture. 
Journal of General Microbiology, Vol. 33, No. 3

Plate 1
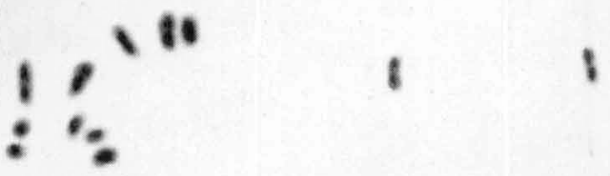

1

2

3
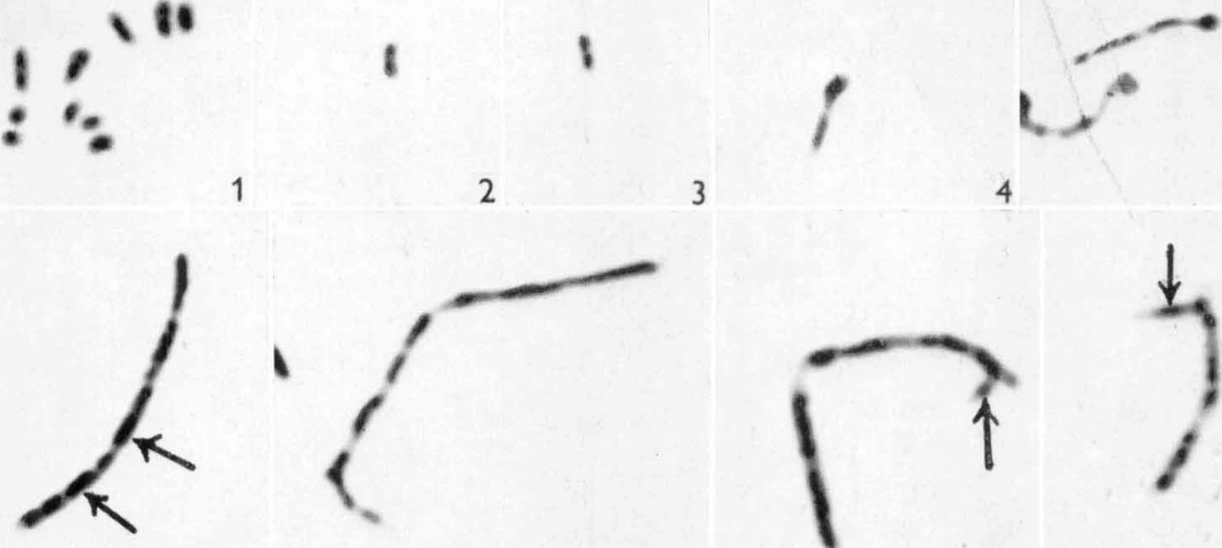

4

5

7

8
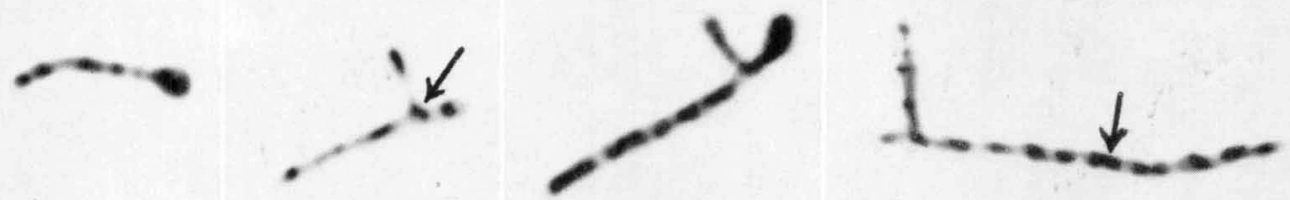

10

11

12

13

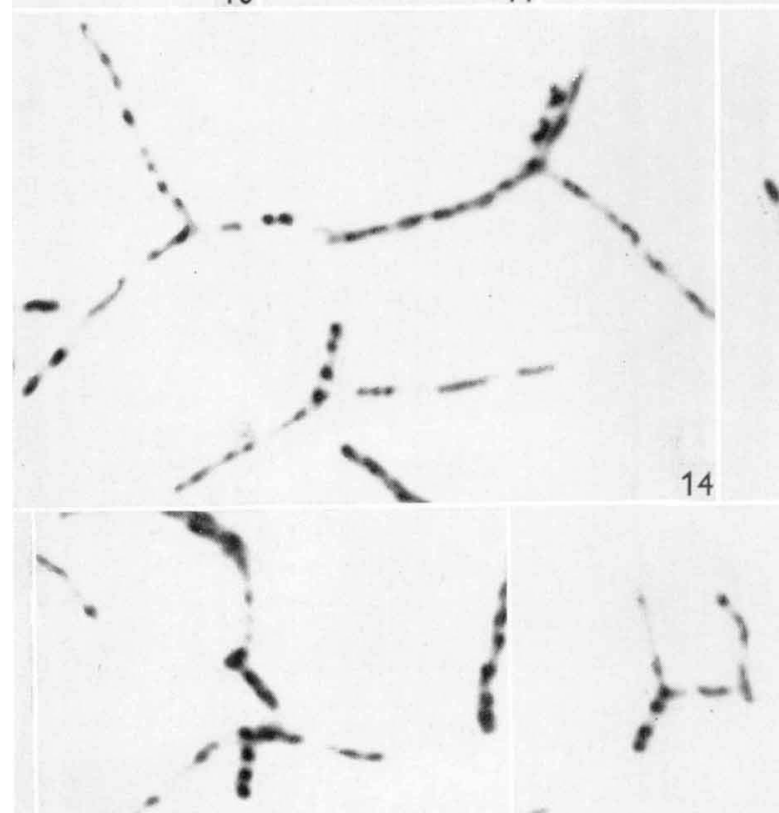

18. 
Journal of General Microbiology, Vol. 33, No. 3

Plate 2

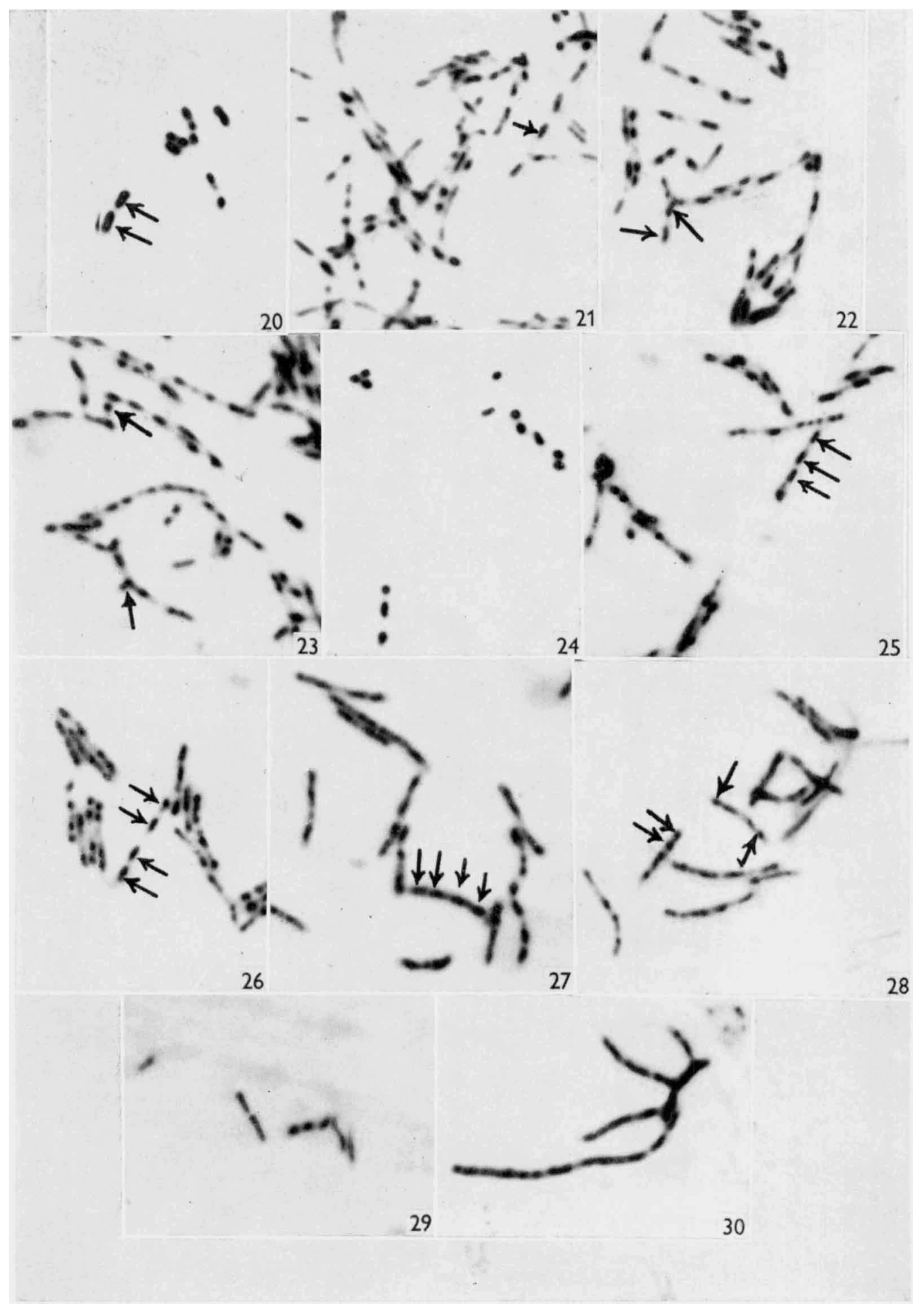

J. N. ADAMS 


\section{Plate 2}

Figs. 20-23. Nocardia erythropolis.

Fig. 20. $3 \mathrm{hr}$. culture. Arrows indicate dumb-bell shaped chromatin bodies.

Fig. 21. $12 \mathrm{hr}$. culture. Arrow indicates median chromatin body division.

Fig. 22. $12 \mathrm{hr}$. culture. Arrows indicate terminal chromatin body division; and chromatin body migration at base of branch.

Fig. 23. $15 \mathrm{hr}$. culture. Arrows indicate chromatin body migrations into new branch and single spherical chromatin body in branch.

Figs. 24-28. Nocardia canicruria.

Fig. 24. $3 \mathrm{hr}$. culture.

Fig. 25. 9 hr. culture. Arrows indicate synchronous chromatin body divisions.

Fig. 26. $12 \mathrm{hr}$. culture. Arrows indicate synchronous chromatin body divisions.

Fig. 27. $18 \mathrm{hr}$. culture. Arrows indicate synchronous chromatin body divisions.

Fig. 28. $21 \mathrm{hr}$. cultures. Early fragmentation; arrows indicate separation of distinct chromatin bodies in cell.

Figs. 29-30. Nocardia asteroides.

Fig. 29. Inoculum.

Fig. 30. 24 hr. culture. 